\title{
ANALYTICAL MODEL OF ELASTIC FRACTURE TOUGHNESS FOR STEEL PIPES WITH INTERNAL CRACKS
}

\author{
Shangtong Yang ${ }^{\mathrm{a}^{*}}$, Chun-Qing $\mathrm{Li}^{\mathrm{b}}$ and Wei Yang ${ }^{\mathrm{c}}$ \\ ${ }^{a}$ Department of Civil and Environmental Engineering, University of Strathclyde, Glasgow, G1 \\ 1XJ, UK \\ ${ }^{\mathrm{b}}$ School of Civil, Environmental and Chemical Engineering, RMIT University, GPO Box 2476, \\ Melbourne 3001, Australia \\ ${ }^{c}$ School of Civil Engineering and Architecture, Wuhan University of Technology, Wuhan, \\ 430070, China
}

\begin{abstract}
Corrosion or manufacture defects can cause internal cracks in steel pipes. For ductile materials, the crack front can yield before the stress intensity reaches its fracture toughness. The yielding of the crack front could ease the stress concentration at the crack front. Therefore, to predict the failure of cracked steel pipes using linear elastic fracture mechanics it is necessary to quantify the part of fracture toughness that withstands the elastic stress field, namely, elastic fracture toughness. This paper intends to propose an analytical model of the elastic fracture toughness for steel pipes with internal surface cracks.
\end{abstract}

\section{KEYWORDS}

Stress intensity factor; Elastic toughness; Weight function; Surface crack; Steel pipes.

* Corresponding author. Tel: +44 141548 3273. Email: shangtong.yang@strath.ac.uk. 


\section{INTRODUCTION}

Pipes are widely used to transport gas or liquid in infrastructure and industries, e.g., water, oil/gas, carbon dioxide etc. These pipes are made of various materials based on different needs amongst which steel is very commonly used. For steel material, there are a number of classes or grades according to the carbon content with different mechanical properties. Most of steel materials exhibit ductile behaviour, demonstrated by yielding of the material. The yielding behaviour allows the steel pipes undergo significant amount of deformation before the collapse which to some extent prevents catastrophic failures. When pipes collapse, however they fail, it can be socially, economically and environmentally devastating, causing, e.g., enormous disruption of daily life, massive costs of reinstatement, widespread flooding and subsequent pollution, and so on.

Like in many other structures, it is inevitable to have defects or cracks in steel pipes. The defects are normally produced during manufacturing process and the cracks are mainly induced by material deterioration, e.g., corrosion. Surface cracks are perhaps the most common form [1] and usually treated as semi-elliptical shape $[2,3]$. The schematic and geometry of the surface crack in a pipe is shown in Figure 1. Due to the presence of surface cracks, the stress in a pipe will concentrate around those cracks, known as stress singularity, which is one of main causes for pipe collapse. For brittle materials, e.g., cast iron, stress is developed around cracks elastically and the stress intensity factor can be determined from elastic fracture mechanics to represent the singularised stress. When the stress intensity factor reaches a critical limit, known as fracture toughness, $K_{I C}$, any extra load will cause the failure of the structure.

When a structure is made of ductile materials, the crack front/tip can yield before the stress intensity factors reaches $K_{I C}$. The yielding of the crack front could ease the stress concentration. As such, at the critical state of fracture, the maximum stress intensity factor, as represented by fracture 
toughness $K_{I C}$, consists of elastic and plastic portions and part of stress singularity (or part of $K_{I C}$ ) is endured by the yielding of the crack front/tip. In other words, to be able to use linear elastic fracture mechanics, the plastic portion should be excluded from the fracture toughness $K_{I C}$ of the ductile materials. How much to be deducted depends on the extent of plastic property of the material, e.g., yield stress. Therefore, for predicting the failure of defected/cracked pipes of ductile materials without modelling the plastic development around the crack front, it is necessary to quantify the elastic fracture toughness in $K_{I C}$ if the linear elastic fracture mechanics is to be used.

Considerable research has been carried out on determining the stress intensity factors for pipes with internal surface cracks and most of it focuses on brittle materials [3-6]. Milne et al. [7] developed an assessment framework for integrity of ductile pipes containing defects. In their method, the effects of material yielding at the crack front on pipes are considered in combination with elastic fracture analysis in a failure assessment diagram. Critical points in the diagram have been identified based on test results with respect to both fracture and yielding failure. The fracture toughness can be experimentally determined by ASTM standard testing method [8] or some numerical approaches, e.g., [9]. For plastic pipes, most research employed elastic-plastic finite element analysis to investigate the fracture response (Crack Tip Opening Displacement) of the cracked pipes [10-12]. The elastic-plastic fracture analysis is necessary in modelling crack propagation but can demand more effort on simulation than elastic fracture analysis, including computational time. For thin-walled pipes with internal surface cracks, it is reasonable not to allow any crack propagation through the thickness of the pipe. Therefore, linear elastic fracture mechanics can still be used in assessing the failure of plastic pipes as long as the elastic fracture resistance can be identified and formulated. A comprehensive literature review (see References) suggest that very little research has been undertaken that addresses the plastic fracture capacity of pipes made of ductile materials and almost none in an analytical manner. Given the fact that fracture mechanics has been widely employed to determine stress intensity factors for both brittle 
and ductile materials, there is a clear need to develop a model for elastic fracture toughness with which the simple failure criterion of linear elastic fracture mechanics could be used in assessing cracked ductile pipes and in the meantime the plastic property of pipe is considered.

This paper proposes an analytical model of elastic fracture toughness for steel pipes with internal surface cracks, as a function of geometric and material parameters, i.e., crack/pipe geometry, fracture toughness and yield stress. Weight function method is employed to enable the analytical derivation of elastic fracture toughness. Weight functions are derived for a wide range of geometries of cracked pipes, i.e., aspect ratios $0.2 \leq a / c \leq 2.0$ and $0.2 \leq a / t \leq 0.8$. With the proposed model the failure criteria of elastic fracture mechanics can be used to assess fracture of ductile materials. Tearing is not considered in the model for failure assessment, which can provide some safe margin for relatively thin-walled structures. An example is provided to demonstrate the application of the proposed model and some key parameters that affect the fracture of carked pipes have been investigated. The proposed analytical model can help engineers and asset managers in risk assessment of pipe failures so as to make a risk informed decision with regards to repair and maintenance of deteriorated steel pipes.

\section{FAILURE CRITERIA}

According to elastic fracture mechanics when a pipe has a crack on its wall, a stress intensity factor is used to assess its fracture as follows:

$K_{I} \geq K_{I C} \quad$ when a pipe fails

$K_{I}$ is the stress intensity factor and $K_{I C}$ is the fracture toughness both for mode I fracture.

For ductile materials, the contribution of the material yielding to the fracture toughness $K_{I C}$ needs to be accounted for. The yielding would provide a plastic phase in stress development which could 
ease the stress intensity due to the dissipation of strain energy by enduring large deformation of the material without increasing the stress. In other words, the stress singularity around a crack can be softened by plastic deformation of the material. Thus using Equation (1) to assess fracture of ductile materials may have neglected their yielding behaviour and hence leads to inaccurate prediction of the failure.

To enable the continual use of linear elastic fracture mechanics, i.e., Equation (1), and in the meantime to account for the softening of stress intensity due to material yielding, a function $K_{I C}^{\prime}$ is proposed such that the failure criteria of Equation (1) becomes

$$
K_{I} \geq K_{I C}^{\prime} \quad \text { when a pipe fails }
$$

where $K_{I C}^{\prime}$ is referred to as elastic fracture toughness in this paper which depends on plastic development of the material. When there is no plastic development $K_{I C}^{\prime}=K_{I C}$. With Equation (2) the elastic fracture mechanics can still be used to determine $K_{I}$ and the plastic property of the material is taken into account by modifying the "full" fracture toughness $K_{I C}$.

For internally pressurized pipes, two parameters measuring two individual failure modes, i.e., fracture or loss of strength, also known as fracture toughness parameter and load parameter in standard FAD analysis, can be introduced as follows:

$$
\begin{aligned}
& K_{r}=\frac{K_{I}}{K_{I C}} \\
& L_{r}=\frac{P}{P_{L}}
\end{aligned}
$$

where $P$ is the applied load/pressure and $P_{L}$ is the plastic limit load/pressure of the cracked pipes. Normally a pipe fails when either $K_{r}$ or $L_{r}$ reaches a certain limit. However, these two failure modes interact. Loss of strength is marked by material yielding which in turn affects the development of stress intensity and hence affects the failure by fracture. Thus it is important to 
understand this interactive behaviour of the two failure mechanisms. Milne and his co-workers have established a quantitative relationship between $K_{r}$ and $L_{r}$ at the critical state through experiments. It can be expressed as follow [7]

$$
\begin{aligned}
& K_{r}=\left(1-0.14 L_{r}^{2}\right)\left[0.3+0.7 \exp \left(-0.65 L_{r}^{6}\right)\right] \\
& L_{r}^{\max }=\frac{\bar{\sigma}}{\sigma_{y}}
\end{aligned}
$$

where $\bar{\sigma}$ is the uniaxial flow stress of the material, obtained as the average of the yield and ultimate tensile strengths. $\sigma_{y}$ is the uniaxial lower yield stress, based on uniaxial tensile test. Equations (5) and (6) are the basis for deriving the elastic fracture toughness $K_{I C}^{\prime}$ as follows.

\section{DIREVATION OF ELASTIC FRACTURE TOUGHNESS}

According to the theory of fracture mechanics [13], the stress intensity factor $K_{I}$ is a function of far-field stress level $\sigma$, the size of the crack $a$ (crack depth in surface cracks), the shape and orientation of the crack, and dimensions of the body, i.e., the pipe wall, in which the crack occurs. This relationship can be expressed in general as follows [14]

$K_{I}=\sigma \sqrt{\pi a} f(\phi)$

where $\phi$ is an angle that defines the positions of the points along the crack front and $f(\phi)$ is the shape function allowing for various geometries of the crack and the pipe. To determine the stress intensity factor $K_{I}$, finite element method (FEM) is usually employed especially for cases where analytical solutions cannot be obtained [15]. Since the stress intensity factors for cracks in a pipeline depend on crack geometries and also vary along the crack front, it is useful to have a general solution applicable to a wide range of geometries at some critical points of the crack. Weight functions are therefore developed to determine stress intensity factor $K_{I}$ which is the product of the distributed stress $\sigma(x)$ along the crack and the corresponding weight function. $\sigma(x)$ 
is the hoop stress on the crack surface along the $\mathrm{x}$ direction which is the thickness direction as illustrated in Figure 1. The stress intensity factor $K_{I}$ can be expressed as follows $[16,17]$

$$
K_{I}=\int_{0}^{a} m(x, a) \sigma(x) d x
$$

where $m(x, a)$ is the weight function of position $x$ and crack depth $a$.

Based on the theory of superposition, the distributed stress $\sigma(x)$ along the crack can be related to the internal pressure $P[18]$ as follows,

$$
\sigma(x)=P+\frac{P R^{2}}{(R+t)^{2}-R^{2}}\left[1+\frac{(R+t)^{2}}{(R+x)^{2}}\right]
$$

where $R$ is the inner radius of the pipe and $t$ is the thickness of the pipe wall as shown in Figure 1.

For internal surface cracks, the plastic limit pressure $P_{L}$ should be considered as the limit internal pressure to yield the extreme fibre of the pipe wall for local failure rather than global collapse of the pipe. With this assumption, a derived form of $P_{L}$ from an empirical model [19] is presented as follows,

$$
P_{L}=\frac{\sigma_{y} t(1-a / t)}{(R+t / 2)(1-\gamma a / t)}
$$

where $\gamma=\left[1+\frac{1.05 c^{2}}{(R+t / 2) t}\right]^{-0.5}$ and $\sigma_{y}$ is the yield strength of the pipe material.

Substituting Equations (8) - (10), Equations (3) and (4) become

$$
\begin{aligned}
& K_{r}=\frac{P \int_{0}^{a} m(x, a)\left\{1+\frac{R^{2}}{(R+t)^{2}-R^{2}}\left[1+\frac{(R+t)^{2}}{(R+x)^{2}}\right]\right\} d x}{K_{I C}} \\
& L_{r}=\frac{P}{\frac{\sigma_{y} t(1-a / t)}{(R+t / 2)(1-\gamma a / t)}}
\end{aligned}
$$


Dividing Equation (11) by Equation (12), $K_{r}$ can be expressed in terms of $L_{r}$ as follows,

$$
K_{r}=\frac{\sigma_{y} t(1-a / t) \int_{0}^{a} m(x, a)\left\{1+\frac{R^{2}}{(R+t)^{2}-R^{2}}\left[1+\frac{(R+t)^{2}}{(R+x)^{2}}\right]\right\} d x}{K_{I C}(R+t / 2)(1-\gamma a / t)} L_{r}
$$

Combining Equations (5) and (13), the critical limit of $K_{r}$ at fracture, denoted as $K_{r c}$, can be obtained as follows,

$$
K_{r c}=\left[1-0.14\left(\frac{K_{r c} K_{I C}(R+t / 2)(1-\gamma a / t)}{\sigma_{y} t(1-a / t) \int_{0}^{a} m(x, a) \beta d x}\right)^{2}\right]\left\{0.3+0.7 \exp \left\{-0.65\left[\frac{K_{r c} K_{I C}(R+t / 2)(1-\gamma a / t)}{\sigma_{y} t(1-a / t) \int_{0}^{a} m(x, a) \beta d x}\right]^{6}\right\}\right\}
$$

where $\beta=1+\frac{R^{2}}{(R+t)^{2}-R^{2}}\left[1+\frac{(R+t)^{2}}{(R+x)^{2}}\right]$

and $1 \geq K_{r c} \geq\left[1-0.14\left(\frac{\bar{\sigma}}{\sigma_{y}}\right)^{2}\right]\left[0.3+0.7 \exp \left(-0.65\left(\frac{\bar{\sigma}}{\sigma_{y}}\right)^{6}\right)\right]$.

From Equation (3), when the critical state of failure is reached, the stress intensity factor of Equation (3), i.e., $K_{I}$, becomes elastic critical limit, i.e., elastic fracture toughness, $K_{I C}^{\prime}$ which can be written as follows,

$$
K_{I C}^{\prime}=K_{r c} K_{I C}
$$

Equation (15) actually modifies the fracture toughness $K_{I C}$ by $K_{r c}$. The elastic fracture toughness $K_{I C}^{\prime}$ is defined as the critical stress intensity factor relating to elastic stress only; this definition is in line with the concept of elastic and plastic stress intensity factors in Milne et al [7]. It needs to be noted that $K_{I C}^{\prime}$ is a constant given the same crack/pipe geometry and material property. This is reflected in Equation (14) where $K_{r c}$ is a function of geometry and material property only. It also needs to be noted that Equation (14) is an implicit expression of $K_{r c}$ and it can be solved by numerical method with, e.g., MatLab. 


\section{WEIGHT FUNCTION}

To determine $K_{I}$ as shown in Equation (8), weight function has to be derived for a given location. For the case of pipes with internal surface cracks, the deepest and surface points of the surface crack are normally of interest as those two points usually have the maximum and minimum stress intensities. The weight functions for the surface point $m_{S}(x, a)$ and the deepest point $m_{D}(x, a)$ can be derived based on finite element analysis results [20] for a wide range of crack geometries, i.e., $0.2 \leq a / c \leq 2.0$ and $0.2 \leq a / t \leq 0.8$ as shown below. These ranges of geometric factors cover most of the practical cases of internally cracked steel pipes.

$m_{S}(x, a)=\frac{2}{\sqrt{\pi x}}\left\{1+\left[\frac{\pi}{\sqrt{Q}}\left(6 G_{0}^{S}-30 G_{1}^{S}\right)-8\right]\left(\frac{x}{a}\right)^{\frac{1}{2}}+\left[\frac{15 \pi}{\sqrt{Q}}\left(6 G_{1}^{S}-G_{0}^{S}\right)+15\right]\left(\frac{x}{a}\right)+\left[\frac{\pi}{\sqrt{Q}}\left(9 G_{0}^{S}-60 G_{1}^{S}\right)-8\right]\left(\frac{x}{a}\right)^{\frac{3}{2}}\right\}$

where $G_{0}^{S}=H_{1}+H_{2}\left(\frac{a}{t}\right)+H_{3}\left(\frac{a}{t}\right)^{2}$

$$
\begin{aligned}
& H_{1}=0.4868+0.4174\left(\frac{a}{c}\right)+0.852\left(\frac{a}{c}\right)^{2}-0.8787\left(\frac{a}{c}\right)^{3}+0.2376\left(\frac{a}{c}\right)^{4} \\
& H_{2}=-0.7856+4.343\left(\frac{a}{c}\right)-7.603\left(\frac{a}{c}\right)^{2}+5.402\left(\frac{a}{c}\right)^{3}-1.287\left(\frac{a}{c}\right)^{4} \\
& H_{3}=2.022-5.949\left(\frac{a}{c}\right)+8.646\left(\frac{a}{c}\right)^{2}-5.798\left(\frac{a}{c}\right)^{3}+1.358\left(\frac{a}{c}\right)^{4} \\
& G_{1}^{S}=H_{4}+H_{5}\left(\frac{a}{t}\right)+H_{6}\left(\frac{a}{t}\right)^{2} \\
& H_{4}=0.0631-0.0924\left(\frac{a}{c}\right)+0.8076\left(\frac{a}{c}\right)^{2}-0.8199\left(\frac{a}{c}\right)^{3}+0.228\left(\frac{a}{c}\right)^{4} \\
& H_{5}=-0.3308+2.46\left(\frac{a}{c}\right)-5.414\left(\frac{a}{c}\right)^{2}+4.515\left(\frac{a}{c}\right)^{3}-1.189\left(\frac{a}{c}\right)^{4}
\end{aligned}
$$




$$
H_{6}=0.7072-2.952\left(\frac{a}{c}\right)+5.738\left(\frac{a}{c}\right)^{2}-4.657\left(\frac{a}{c}\right)^{3}+1.219\left(\frac{a}{c}\right)^{4}
$$

$Q$ is an elliptical integral of the second kind (which defines the shape of the ellipse) and can be expressed empirically as follows [21]

$$
\begin{array}{ll}
Q=1+1.464(a / c)^{1.65} & \text { for } a / c \leq 1 \\
Q=\left\lfloor 1+1.464(c / a)^{1.65}\right\rfloor(a / c)^{2} & \text { for } a / c>1
\end{array}
$$

Likewise,

$$
m_{D}(x, a)=\frac{2}{\sqrt{2 \pi(a-x)}}\left\{1+\left[\frac{3 \pi}{5 \sqrt{2 Q}}\left(\frac{2}{3} G_{0}^{D}-2 G_{1}^{D}\right)+1\right]\left(1-\frac{x}{a}\right)^{\frac{1}{2}}+3\left(1-\frac{x}{a}\right)+\left[\frac{6 \pi}{5 \sqrt{2 Q}}\left(G_{0}^{D}+2 G_{1}^{D}\right)-10\right]\left(1-\frac{x}{a}\right)^{\frac{3}{2}}\right\}
$$

where $G_{0}^{D}=F_{1}+F_{2}\left(\frac{a}{t}\right)+F_{3}\left(\frac{a}{t}\right)^{2}$

$$
\begin{aligned}
F_{1} & =0.9569+0.1599\left(\frac{a}{c}\right)-0.2225\left(\frac{a}{c}\right)^{2}+0.1245\left(\frac{a}{c}\right)^{3}-0.0243\left(\frac{a}{c}\right)^{4} \\
F_{2} & =0.7177-1.056\left(\frac{a}{c}\right)+0.3893\left(\frac{a}{c}\right)^{2}+0.1081\left(\frac{a}{c}\right)^{3}-0.061\left(\frac{a}{c}\right)^{4} \\
F_{3} & =1.901-7.734\left(\frac{a}{c}\right)+10.8\left(\frac{a}{c}\right)^{2}-6.173\left(\frac{a}{c}\right)^{3}+1.231\left(\frac{a}{c}\right)^{4} \\
G_{1}^{D}=F_{4}+F_{5}\left(\frac{a}{t}\right)+F_{6}\left(\frac{a}{t}\right)^{2} & \\
F_{4} & =0.596+0.2935\left(\frac{a}{c}\right)-0.5407\left(\frac{a}{c}\right)^{2}+0.5088\left(\frac{a}{c}\right)^{3}-0.1424\left(\frac{a}{c}\right)^{4} \\
F_{5} & =0.3378-1.81\left(\frac{a}{c}\right)+3.886\left(\frac{a}{c}\right)^{2}-3.331\left(\frac{a}{c}\right)^{3}+0.8971\left(\frac{a}{c}\right)^{4} \\
F_{6} & =0.6076-1.193\left(\frac{a}{c}\right)-0.1731\left(\frac{a}{c}\right)^{2}+1.392\left(\frac{a}{c}\right)^{3}-0.5382\left(\frac{a}{c}\right)^{4}
\end{aligned}
$$




\section{VERIFICATION AND DISCUSSIONS}

Before verifying the developed model for elastic toughness, an example is presented to demonstrate its application to practical steel pipes. Most pipes are subjected to internal pressure, e.g., pressurised water, gas or liquid-phase carbon dioxide, which produce stresses in the wall of the pipe. The material properties for the steel in the example are taken as $\sigma_{y}=400 \mathrm{MPa}, \bar{\sigma}=650$ $M P a$ and $K_{I C}=100 \mathrm{MPa} / \sqrt{\mathrm{m}}$. The geometry of the pipeline with internal surface crack is taken as $a / c=2.0, a / t=0.5, t / R=0.1$ and $a=0.1 \mathrm{~m}$. The internal pressure is assumed to be 100 $M P a$. The process to determine both the stress intensity factor, i.e., Equation (8), and the elastic fracture toughness, i.e., Equation (15), is as follows.

The first is to evaluate the weight functions for the surface and deepest points, i.e., Equations (16) and (19) and these two weight functions become:

$$
\begin{aligned}
& m_{S}(x, a)=\frac{2}{\sqrt{\pi x}}\left[1-4.05 \times\left(\frac{x}{a}\right)^{1 / 2}+9.25 \times\left(\frac{x}{a}\right)-6.20 \times\left(\frac{x}{a}\right)^{3 / 2}\right] \\
& m_{D}(x, a)=\frac{2}{\sqrt{2 \pi(a-x)}}\left[1+0.463 \times\left(1-\frac{x}{a}\right)^{1 / 2}+3 \times\left(1-\frac{x}{a}\right)-7.09 \times\left(1-\frac{x}{a}\right)^{3 / 2}\right]
\end{aligned}
$$

Substituting the above evaluated weight functions together with the stress distribution expressions (Equation (9)) into Equation (8), the stress intensity factor can be obtained as follows:

$K_{I}=412 M P a \sqrt{m}$ for the surface point and,

$K_{I}=255 M P a \sqrt{m}$ for the deepest point.

Next is to determine the elastic fracture toughness. Substituting the derived weight functions into Equation (14), $K_{r c}$ can be obtained as $K_{r c}=0.93$. From Equation (15), the elastic fracture toughness can then be calculated, i.e., $K_{I c}^{\prime}=93 M P a \sqrt{m}$. 
Finally, Equation (2) is used to assess the failure of the pipes. For this example, since $K_{I}>K_{I c}^{\prime}$, the pipe failed.

To verify the developed model of Equation (15), it would be ideal to have experimental results for the elastic fracture toughness directly but this proves to be extremely difficult from literature search. However, some results have been found on $K_{r}$ which can be used to compare with those from the analytical model for indirect verification. Amongst the test data published [22, 23], most are related to very small ratios of $a / c$ or $a / t$, e.g., $a / c=0.02$. They do not fall in the applicable ranges of the derived weight function and the model cannot be directly used to derive $K_{r}$. Brown and Zybenko [23] and Schulze et al. [22] have tested a number of cylindrical pipes with artificial axial flaws to different depths/geometries, measured the pressure at failure and calculated the stress intensity factors. Their results were summarized in Miller [19] from which the data were obtained for comparisons as presented in Table 1. By using the same inputs of geometries and pressures, $K_{r}$ for the deepest point determined from the proposed model (Equation (13)) and the literature are in reasonably good agreement. The difference in the results could be caused by possible variation in formulation of the stress intensity factor $K_{I}$, since the same value of $K_{I C}$ is used.

One of the advantages of the proposed model is that the effects of some key parameters on fracture of the pipes can be quantitatively investigated. From Equation (14), it can be found that it is not simply the yield strength or the critical stress intensity factor, but the ratio of them that controls the elastic fracture toughness as represented by $K_{r c}$, given the same pipe and crack geometries. Figure 2 demonstrates the effect of $K_{I C} / \sigma_{y}$ ratio, a material constant, on the elastic fracture toughness. A range of values of $K_{I C} / \sigma_{y}$ are investigated between 0 and 1 , and the unit of $K_{I C} / \sigma_{y}$ is $\sqrt{m}$. It is very interesting to observe that a higher ratio of $K_{I C} / \sigma_{y}$ leads to a greater portion of fracture resistance being endured by plastic deformation. In general, $K_{I C}$ is a measure of fracture toughness 
and $\sigma_{y}$ is a measure of strength and the ratio $K_{I C} / \sigma_{y}$ reflects an important mechanical property, i.e., toughness/strength. Figure 2 presents a clear indication as to how much amount of fracture resistance is sustained by plastic deformation.

By using Equations (5) and (6), $K_{r}-L_{r}$ curves can be plotted for parametric study. For given applied internal pressures, $K_{r}$ and $L_{r}$ can be calculated by Equations (3), (4), (9) and (16) - (20) for the surface and deepest points respectively as shown in Figure 3. It can be seen from Figure 3 that for a pressure of $10 \mathrm{MPa}$, the stress intensity factor at the deepest point exceeds the critical limit while that at the surface point does not. It has also been observed that, when internal pressure increases, $K_{r}$ linearly increases with $L_{r}$ by the same slope. The linearity is because both $K_{r}$ and $L_{r}$ are linear functions of the applied pressure and thus $K_{r} / L_{r}$ is a constant, i.e., the slope of the straight line in Figure 3. The slopes however depend on the crack geometries, fracture toughness and yield strength. For example, from Equation (10), one can find that smaller yield strength $\sigma_{y}$, produces smaller plastic limit pressure $P_{L}$ and larger $L_{r}$ (Equation (12)). Thus the slope of $K_{r} / L_{r}$ is smaller as shown in Figure 3. This makes sense noting the fact that the smaller the yield strength is the easier the pipe fails.

The effect of fracture toughness of the material on its failure can be investigated through the derived analytical model, given the same strength, i.e., $\sigma_{y}$. Figure 4 demonstrates the $K_{r}-L_{r}$ lines for $K_{I C}=50,75$ and $100 \mathrm{MPa} / \sqrt{\mathrm{m}}$ respectively and the critical curve for failure. The other parameters for all these three lines are the same, i.e., $a / c=0.2, a / t=0.2$ and $a=0.02 m$. It can be seen from Figure 4 that the greater the fracture toughness of the material is, the larger portion of plastic endurance is from $K_{I C}$ in pipe failure. This means that the pipe will fail in more plastic manner with more plastic deformation ahead of the crack. Given the case demonstrated in Figure 4, for materials with $K_{I C}$ greater than $50 \mathrm{MPa} / \sqrt{\mathrm{m}}$, there is considerable plastic endurance, e.g., 
about $20 \%$ for $K_{I C}=75 \mathrm{MPa} / \sqrt{\mathrm{m}}$ and $33 \%$ for $K_{I C}=100 \mathrm{MPa} / \sqrt{\mathrm{m}}$ at failure. These results effectively justify the need to consider the plastic yielding at the crack front in the analysis of fracture.

Figures 5 and 6 show the effects of $a / c$ ratio on the $K_{r}-L_{r}$ curve for both the deepest and surface points with inputs of $a=0.1 \mathrm{~m}, a / t=0.5$ and $K_{I C}=50 \mathrm{MPa} / \sqrt{\mathrm{m}}$. As can be seen, when $a / \mathrm{c}$ ratio increases from 0.5 to 2.0 the slope of $K_{r}-L_{r}$ line decreases, meaning that the crack with higher aspect ratio of $a / c$ fails with more plastic development at the crack front. It should be noted that for the surface point in Figure 6, the lines for $a / c$ ratio of 0.5 and 1.0 are very close. The amount of plasticity that develops at failure can be reflected by the applied pressure $P$ and the plastic limit pressure $P_{L}$ of the given cracked pipe, i.e., Equation (4). The values of $P_{L}$ have been checked for those three cases to be 45.5 $\mathrm{MPa}, 47.0 \mathrm{MPa}$ and $47.5 \mathrm{MPa}$ for $a / c=0.5,1.0$ and 2.0 respectively. As noted the effect of $a / c$ ratio on the plastic limit pressure $P_{L}$ is minor. However, based on Equation (4), it can be found that the effect of $a / c$ ratio on the maximum allowed applied pressure at fracture is relatively significant, e.g., $P$ is nearly doubled for $a / c$ from 0.5 to 2.0 for respective values of $L_{r}$ of 0.19 and 0.41 for the deepest point. It is very interesting to find that higher aspect ratio of $a / c$ results in higher allowable pressure at failure. In other words, given all the other parameters the same for the same steel pipe under the same amount of applied pressure, the pipes with low aspect ratio can fail much earlier than those with high aspect ratio. This finding can be of practical significance when assessing the pipe failure on site.

To investigate the effects of $a / t$ ratio on the failure mode of cracked pipes, Figures 7 and 8 are produced for $a / t$ ratio of $0.2,0.5$ and 0.8 for the deepest and surface points respectively. In both curves, when $a / t$ ratio increases, the failure of the pipe becomes more brittle with an increased slope of the $K_{r}-L_{r}$ line. In other words, the smaller the $a / t$ ratio is, the more plastic deformation 
is developed around the crack. This makes sense because smaller $a / t$ ratio means more remaining fibres of the pipe wall ahead of the crack and thus more plastic deformation can develop. Moreover, it is interesting to find out that for higher $a / t$ ratios, e.g., $a / t$ equal to 0.5 and 0.8 , the $K_{r}-L_{r}$ lines are not sensitive to plastic development as they are quite close to each other. This means that the pipes with higher ratio of $a / t$ fails at more or less the same level of plasticity.

\section{CONCLUSIONS}

An analytical model of elastic fracture toughness for steel pipes with internal surface cracks has been proposed, as a function of geometric and material properties. With the proposed model the failure criteria of elastic fracture mechanics can be used to assess fracture of ductile materials. Weight function method has been employed to enable the analytical derivation of elastic fracture toughness. After verifying the proposed model indirectly with data published in literature some key parameters that affect the fracture of carked pipes have been investigated. It has been found that, given the same yield strength, the greater the fracture toughness of the material is, the more plastic development there is and that the stress intensity factor at the deepest point exceeds the critical limit faster than that at the surface point. It has also been found that the pipes with cracks of higher aspect ratio of $a / c$ fail with more plastic development at the crack front and that the smaller the $a / t$ ratio is, the more plastic deformation is developed at the crack front. It can be concluded that the proposed analytical model for elastic fracture toughness can assess the fracture of cracked pipes with ductile materials more accurately. Accurate assessment of pipe failures can help engineers and asset managers in making a risk informed decision with regards to repair and maintenance of deteriorated steel pipes. 


\section{ACKNOWLEDGMENTS}

Financial support from Scottish Funding Council for early career researchers exchanges and from Australian Research Council under DP140101547 and LP150100413 is gratefully acknowledged. 


\section{REFERENCES}

[1] Chen Y, Lambert S. Numerical modeling of ductile tearing for semi-elliptical surface cracks in wide plates. International Journal of Pressure Vessels and Piping. 2005;82:417-26.

[2] Diamantoudis AT, Labeas GN. Stress intensity factors of semi-elliptical surface cracks in pressure vessels by global-local finite element methodology. Engineering Fracture Mechanics. 2005;72:1299-312.

[3] Raju I, Newman J. Stress-intensity factors for internal and external surface cracks in pipes. ASME Journal of Pressure Vessels Technology. 1982;104:293-8.

[4] Sethuraman R, Reddy G, Ilango IT. Finite element based evaluation of stress intensity factors for interactive semi-elliptic surface cracks. International Journal of Pressure Vessels and Piping. 2003;80:843-59.

[5] Fett T. Estimation of stress intensity factors for semi-elliptical surface cracks. Engineering Fracture Mechanics. 2000;66:349-56.

[6] Wang X, Lambert SB. Stress intensity factors and weight functions for high aspect ratio semi-elliptical surface cracks in finite-thickness plates. Engineering Fracture Mechanics. 1997;57:13-24.

[7] Milne I, Ainsworth RA, Dowling AR, Stewart AT. Assessment of the integrity of structures containing defects. International Journal of Pressure Vessels and Piping. 1988;32:3-104.

[8] E1820 A. Standard Test Method for Measurement of Fracture Toughness. Pennsylvania, United States: ASTM International; 2013.

[9] Shibanuma K, Aihara S. Quantitative Prediction of Cleavage Fracture Toughness of Ferrite Steel without Adjustable Parameters. Procedia Materials Science. 2014;3:1238-43.

[10] Jayadevan KR, Østby E, Thaulow C. Fracture response of pipelines subjected to large plastic deformation under tension. International Journal of Pressure Vessels and Piping. 2004;81:771-83.

[11] Zhang YM, Yi DK, Xiao ZM, Huang ZH, Kumar SB. Elastic-plastic fracture analyses for pipeline girth welds with 3D semi-elliptical surface cracks subjected to large plastic bending. International Journal of Pressure Vessels and Piping. 2013;105-106:90-102.

[12] Østby E, Jayadevan KR, Thaulow C. Fracture response of pipelines subject to large plastic deformation under bending. International Journal of Pressure Vessels and Piping. 2005;82:201-15.

[13] Ewalds HL, Wanhill RJH. Fracture mechanics. London: Edward Arnold; 1984.

[14] Hertzberg RW. Deformation and fracture mechanics of engineering materials: John Wiley and Sons; 1996.

[15] Tada H, Paris PC, Irwin GR. The stress analysis of cracks handbook. New York: American Society of Mechanical Engineers; 2000.

[16] Bueckner HF. A novel principle for the computation of stress intensity factors. ZAMM. 1970;50:529-45.

[17] Bueckner HF. Weight functions for the notched bar. ZAMM. 1971;51:97-109.

[18] Li CQ, Yang ST. Stress intensity factors for high aspect ratio semi-elliptical internal surface cracks in pipes. International Journal of Pressure Vessels and Piping. 2012;96-97:13-23.

[19] Miller AG. Review of test results for ductile failure pressure of cracked spherical and cylindrical pressure vessels. UK: CEGB; 1984.

[20] Yang S, Ni YL, Li C-Q. Weight function method to determine stress intensity factor for semi-elliptical crack with high aspect ratio in cylindrical vessels. Engineering Fracture Mechanics. 2013;109:138-49.

[21] Shiratori M, Niyoshi T, Tanikawa K. Analysis of stress intensity factors surface cracks subjected to arbitrarily distributed surface stresses. In: Murakami Y, editor. Stress intensity factors handbook. US: Pergamon; 1987. p. 698-727.

[22] Schulze HD, Togler G, Bodmann E. Fracture mechanics analysis on the initiation and propagation of circumferential and longitudinal cracks in straight pipes and pipe bends. Nuclear Engineering and Design. 1980;58:19-31.

[23] Brown KR, Zybenko B. The fracture mechanics of aluminium-alloy gas cylinders. Engineering Fracture Mechanics. 1981;15:1-20. 


\section{LIST OF TABLES}

1. Comparison of $K_{r}$ for the deepest point between the proposed model and those from literature 
Table 1 Comparison of $K_{r}$ for the deepest point between the proposed model and those from literature

\begin{tabular}{c|c|c|c|c|c|c|c|c|c|c}
\hline \hline Material & $\begin{array}{l}\mathrm{R} \\
(\mathrm{mm})\end{array}$ & $\begin{array}{c}a \\
(\mathrm{~mm})\end{array}$ & $a / c$ & $a / t$ & $\begin{array}{c}\mathrm{P} \\
(\mathrm{MPa})\end{array}$ & $\begin{array}{c}K_{I C} \\
(\mathrm{MPa} / \sqrt{m})\end{array}$ & $\begin{array}{c}K_{r} \\
{[23]}\end{array}$ & $\begin{array}{c}K_{r} \\
{[22]}\end{array}$ & $\begin{array}{c}K_{r} \text { (the } \\
\text { model })\end{array}$ & Difference \\
\hline $\begin{array}{c}\text { Aluminum } \\
\text { Steel }\end{array}$ & 100 & 4.4 & 0.27 & 0.40 & 34 & 30 & 1.72 & n.a. & 1.60 & $6.9 \%$ \\
& 1.7 & 0.20 & 0.53 & 38 & 200 & n.a. & 0.30 & 0.27 & $10 \%$ \\
\hline \hline
\end{tabular}




\section{LIST OF FIGURES}

1. A pipe with a semi-elliptical internal surface crack

2. Effects of $K_{I C} / \sigma_{y}$ on the elastic fracture toughness

3. Failure assessment curve for $a / c=0.2, a / t=0.2, a=0.02 \mathrm{~m}$ and $K_{I C}=50 \mathrm{MPa} / \sqrt{\mathrm{m}}$ for the surface and deepest points

4. Effects of critical fracture toughness on failure assessment of the surface point

5. Effects of $a / c$ ratio on failure mode at the deepest point

6. Effects of $a / c$ ratio on failure mode at the surface point

7. Effects of $a / t$ ratio on failure mode at the deepest point

8. Effects of $a / t$ ratio on failure mode of the surface point 


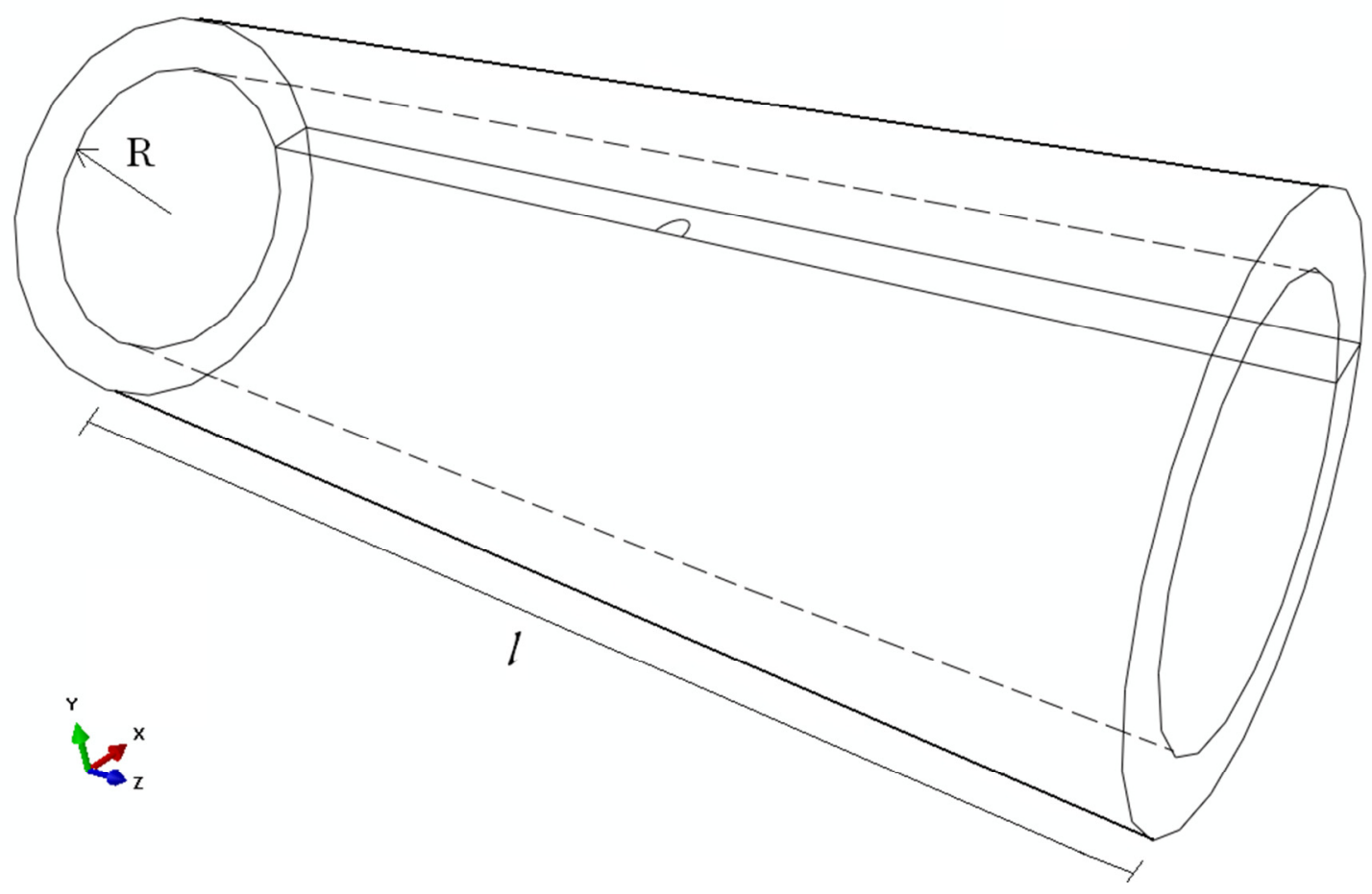

(a) Schematic of a pipe with a semi-elliptical internal surface crack

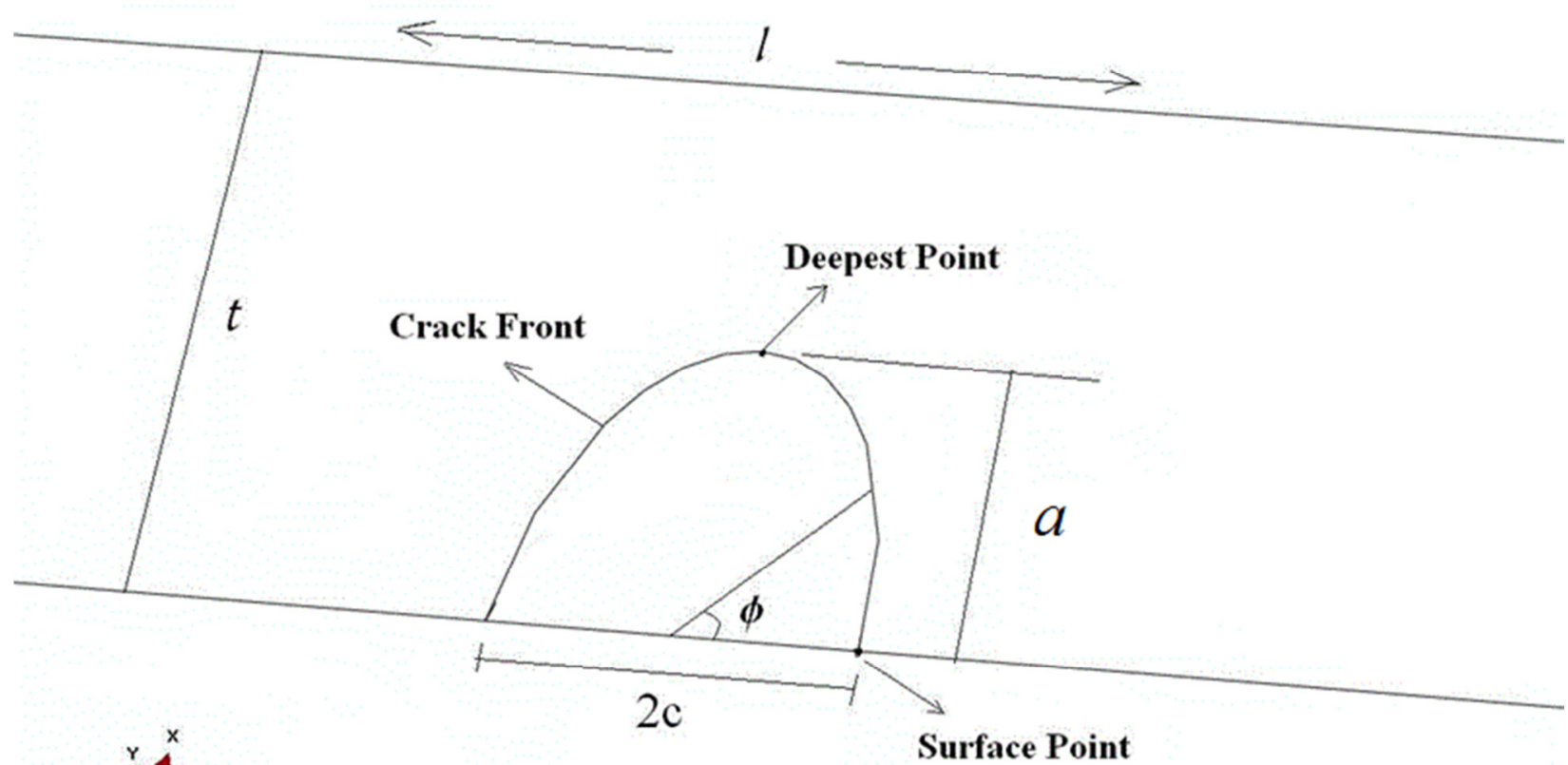

(b) Geometry of the semi-elliptical internal surface crack

Figure 1 A pipe with a semi-elliptical internal surface crack 


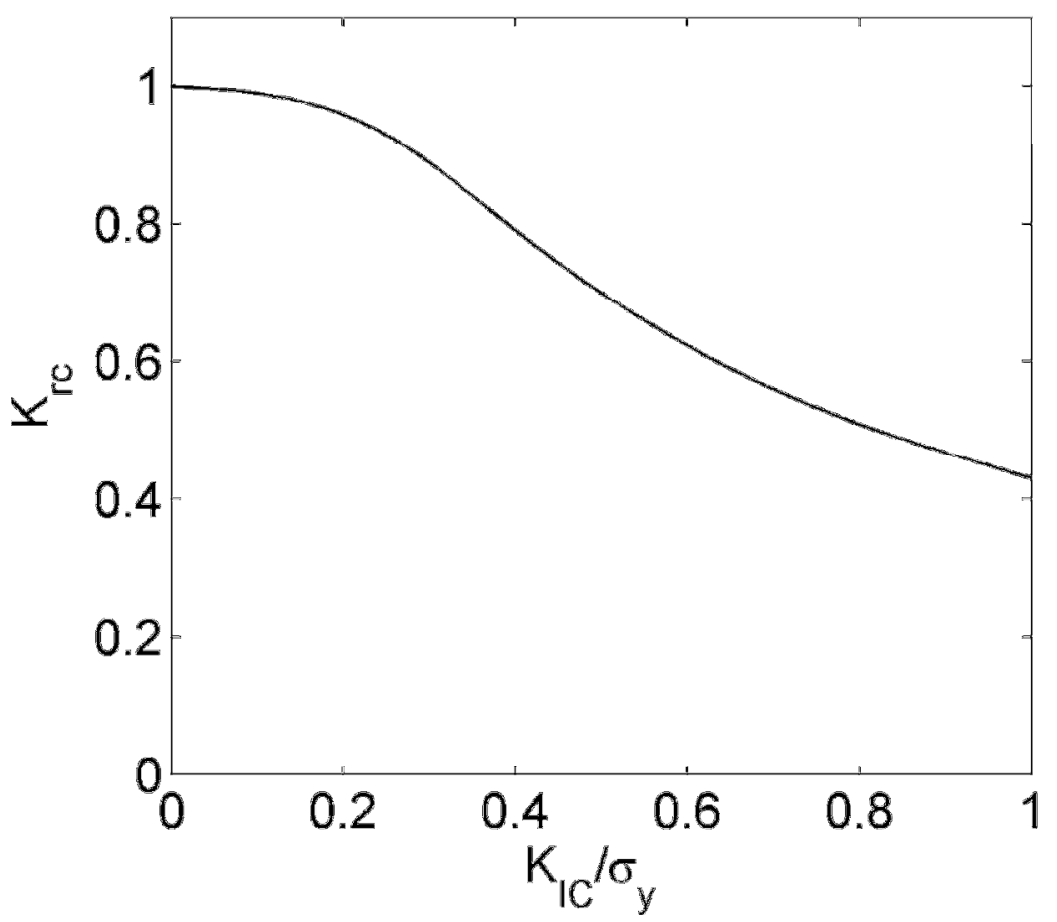

Figure 2 Effects of $K_{I C} / \sigma_{y}$ on the $K_{r c}$ 


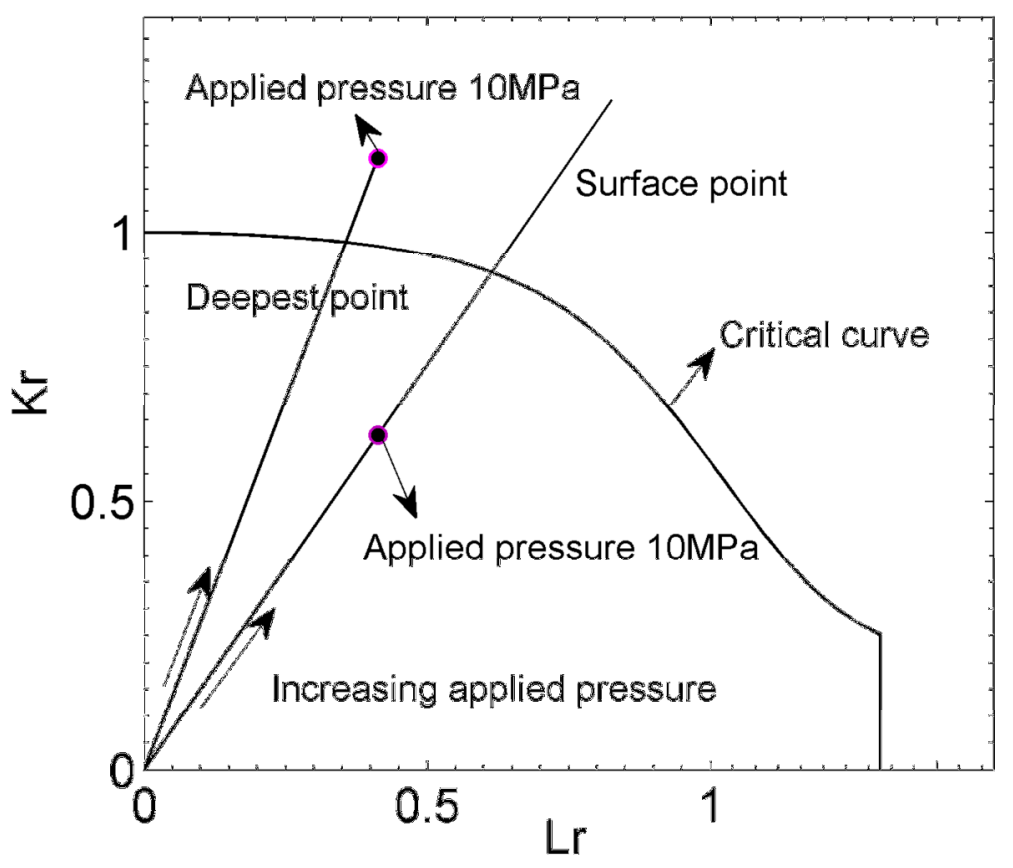

Figure 3 Failure assessment diagram for $a / c=0.2, a / t=0.2, a=0.02 m$ and $K_{I C}=50 \mathrm{MPa} / \sqrt{\mathrm{m}}$ for the surface and deepest points 


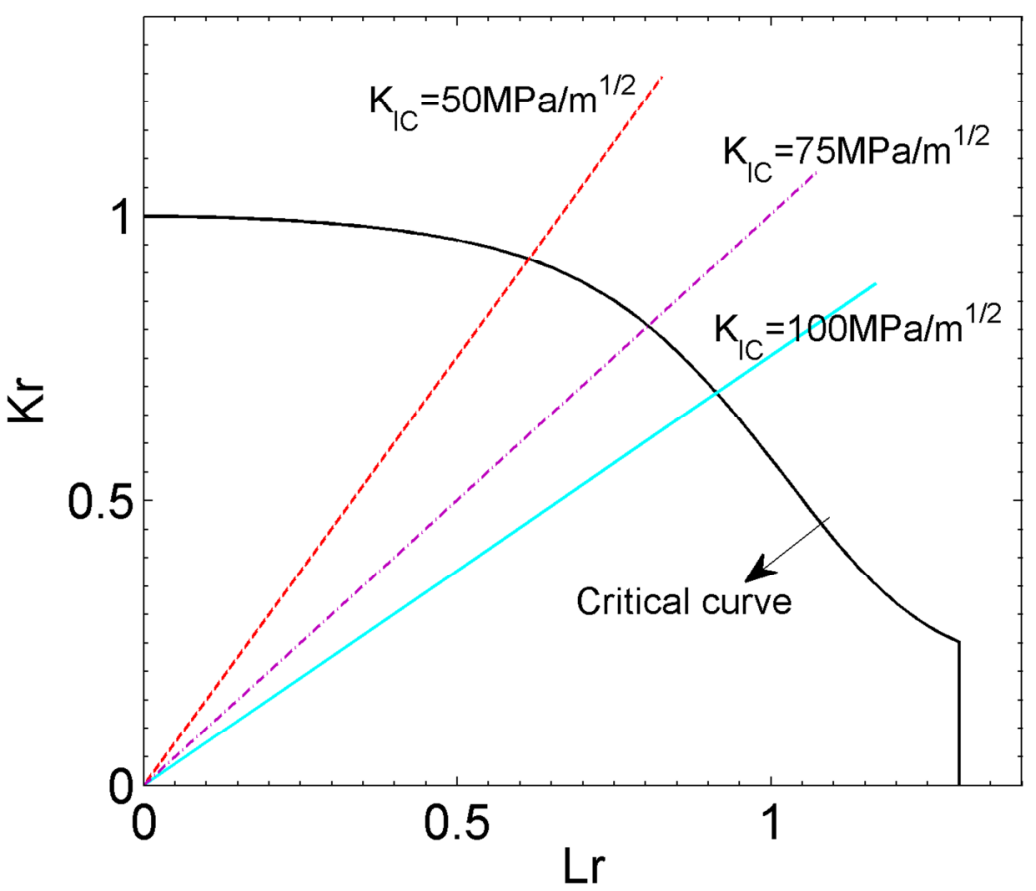

Figure 4 Effects of critical fracture toughness on failure assessment of the surface point 


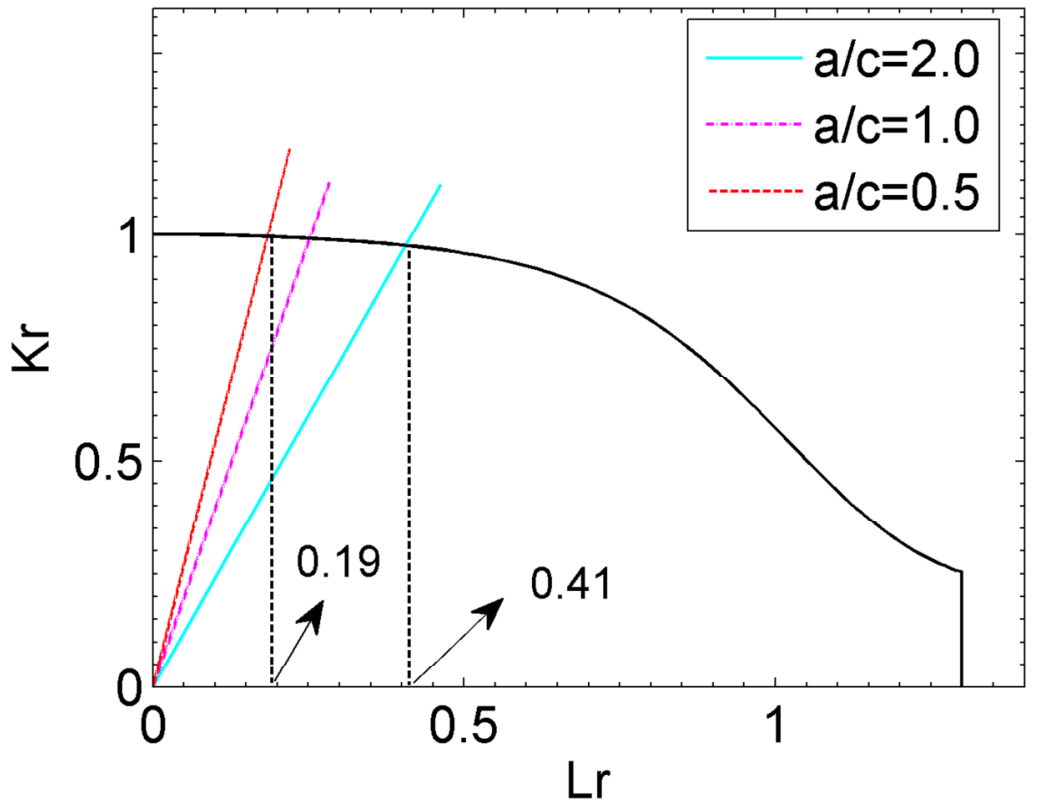

Figure 5 Effects of $a / c$ ratio on failure mode at the deepest point 


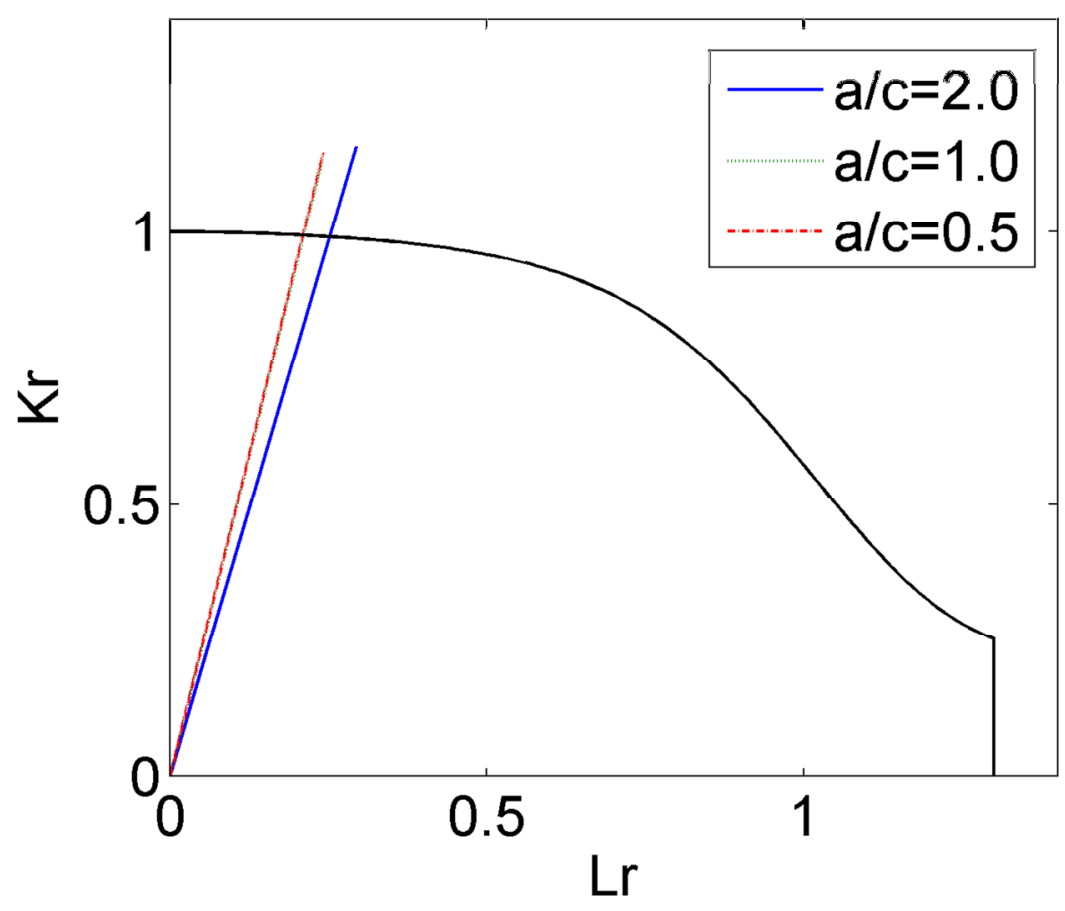

Figure 6 Effects of $a / c$ ratio on failure mode at the surface point 


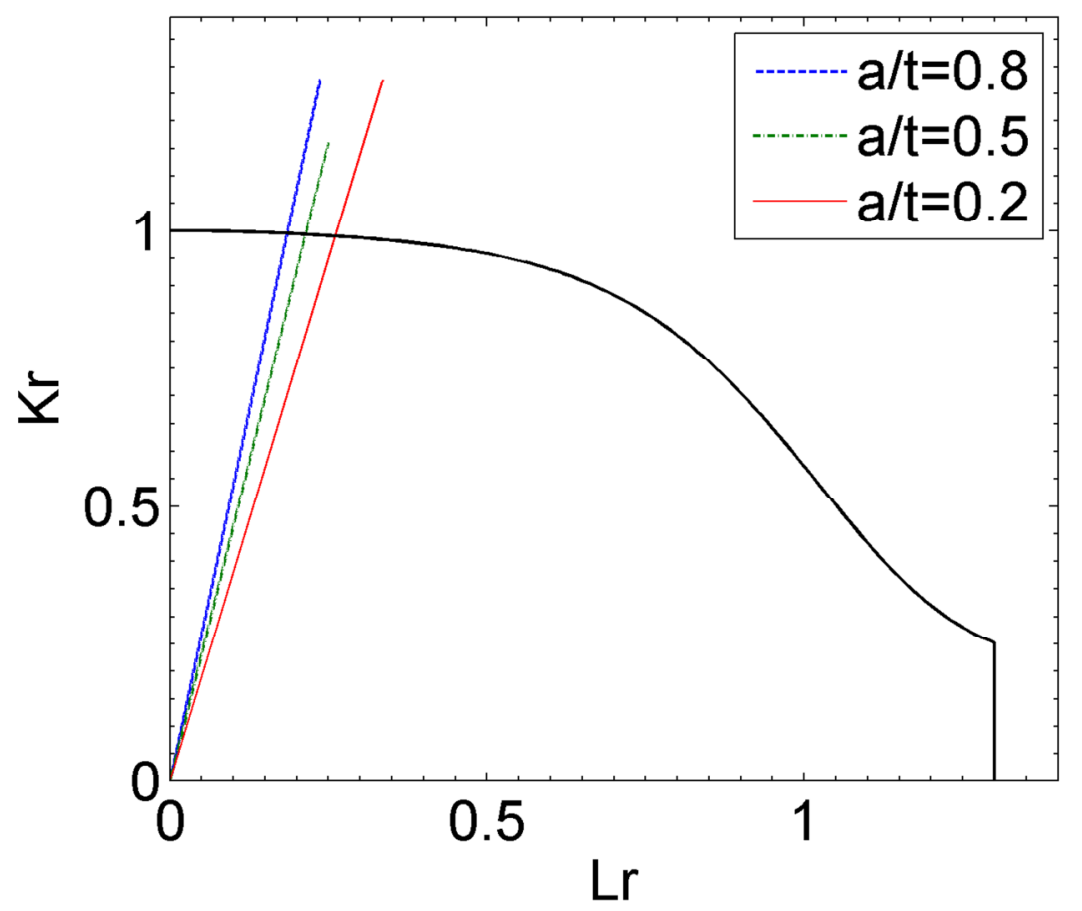

Figure 7 Effects of $a / t$ ratio on failure mode at the deepest point 


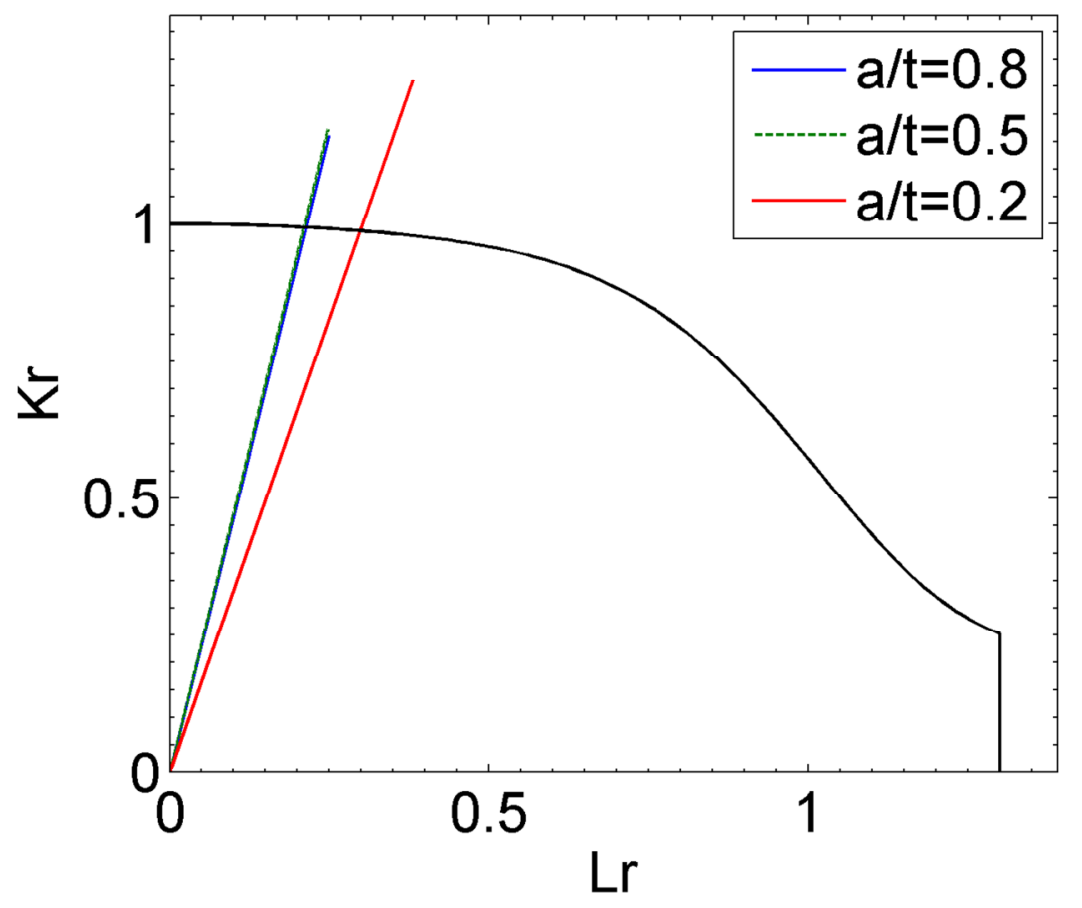

Figure 8 Effects of $a / t$ ratio on failure mode of the surface point 